\title{
Anaplastic lymphoma kinase-positive squamous cell carcinoma of the lung: A case report
}

\author{
YOKO YAMAMOTO $^{1}$, KEN KODAMA ${ }^{1}$, TOMOHIRO MANIWA ${ }^{1}$, MASASHI TAKEDA ${ }^{2}$ and HIROKI KISHIMA ${ }^{3}$ \\ Departments of ${ }^{1}$ Thoracic Surgery and ${ }^{2}$ Pathology, Yao Municipal Hospital; \\ ${ }^{3}$ Department of Surgery, Kishima Hospital Main Division, Yao, Osaka 581-0069, Japan
}

Received January 15, 2016; Accepted April 8, 2016

DOI: $10.3892 /$ mco.2016.878

\begin{abstract}
It is widely known that echinoderm microtubule-associated protein-like 4 anaplastic lymphoma kinase (EML4-ALK) rearrangement mostly occurs in the adenocarcinoma subtype of non-small-cell lung cancer (NSCLC). Patients with squamous cell carcinoma harboring the ALK rearrangement are extremely rare. This is a case report of a squamous cell carcinoma patient with EML4-ALK rearrangement. An elderly man with a heavy smoking history presented with a mass lesion in the right main bronchus. Bronchoscopic biopsy of the tumor confirmed a diagnosis of squamous cell carcinoma, and it was proven to harbor ALK rearrangement, based on fluorescence in situ hybridization, but not epidermal growth factor receptor mutations. The patient underwent radiation therapy, with a markedly favorable response. ALK-targeted treatment may be a viable option if disease progression occurs in such a case in the future.
\end{abstract}

\section{Introduction}

The echinoderm microtubule-associated protein-like 4-ALK (EML4-ALK) fusion gene is a known oncogenic driver in non-small-cell lung cancer (NSCLC), with ALK-translocated NSCLCs accounting for $5 \%$ of all NSCLCs and $20 \%$ of cases in never-smokers (1). This fusion gene is also associated with younger age and the adenocarcinoma subtype of NSCLC. As cases with squamous cell carcinoma of the lung harboring ALK gene rearrangement are extremely rare (2), its molecular analysis is not routinely recommended by the National Comprehensive Cancer Network (NCCN) guideline for the treatment of NSCLC (version 2, 2013) (https://www.tri-kobe. org/nccn/guideline/lung/english/non_small.pdf). We herein describe a rare case of an elderly male smoker with squamous cell carcinoma of the lung harboring ALK rearrangement.

Correspondence to: Dr Yoko Yamamoto, Department of Thoracic Surgery, Yao Municipal Hospital, 1-3-1 Ryuge-Cho, Yao, Osaka 581-0069, Japan

E-mail: yokes615@yahoo.co.jp

Key words: lung cancer, squamous cell carcinoma, anaplastic lymphoma kinase

\section{Case presentation}

A 76-year-old Japanese man was referred to Yao Municipal Hospital due to dyspnea and weight loss. The patient required oxygen supplementation. The past medical history was unremarkable, apart from smoking history (20 pack-years). The serum levels of cytokeratin-19 fragment and carcinoembryonic antigen were elevated to $3.9 \mathrm{ng} / \mathrm{ml}$ (normal, $<3.5 \mathrm{ng} / \mathrm{m}$ ) and $65.8 \mathrm{ng} / \mathrm{ml}$ (normal, <5.0 ng/ml), respectively. A chest radiograph demonstrated right upper lobe atelectasis. Computed tomography $(\mathrm{CT})$ revealed a $26-\mathrm{mm}$ nodule in the right main bronchus and multiple enlarged mediastinal lymph nodes (Fig. 1). Magnetic resonance imaging of the brain revealed no brain metastasis. A pathological diagnosis of squamous cwell carcinoma was made using bronchoscopic biopsy (Fig. 2A). There was no component of adenocarcinoma or other histological type in the biopsy specimen. To further determine the histological subtype, we performed immunohistochemistry (IHC), which was positive for p40 and negative for thyroid transcription factor-1 and napsin A (Fig. 2B-D). Molecular analysis revealed wild-type epidermal growth factor receptor gene status. Although ALK testing using IHC (the iAEP method, ALK detection kit; Nichirei Bioscience, Tokyo, Japan) was negative, fluorescence in situ hybridization analysis with break-apart probes for the ALK gene (Vysis Abbott Molecular, Des Plaines, IL, USA) revealed the presence of an ALK rearrangement (Fig. 3). Based on these results, the patient was clinically diagnosed with T3N2M0, stage IIIA squamous cell carcinoma. The patient had an Easter Cooperative Oncology Group performance status score of 2, and received radiation therapy to the primary site and the mediastinum at a dose of $60 \mathrm{~Gy}$ in 30 fractions. A CT scan at 4 months post-treatment revealed improvement of the atelectasis, with a marked decrease in the size of the tumor. The patient is being regularly followed-up as an outpatient, without oxygen supplementation.

\section{Discussion}

The EML4-ALK fusion gene has been identified as a potent oncogenic driver in NSCLC. ALK-translocated NSCLCs account for $\sim 5 \%$ of all NSCLCs and $20 \%$ of NSCLCs in never-smokers (1). ALK rearrangement has been associated with several clinicopathological characteristics: Never- or light 


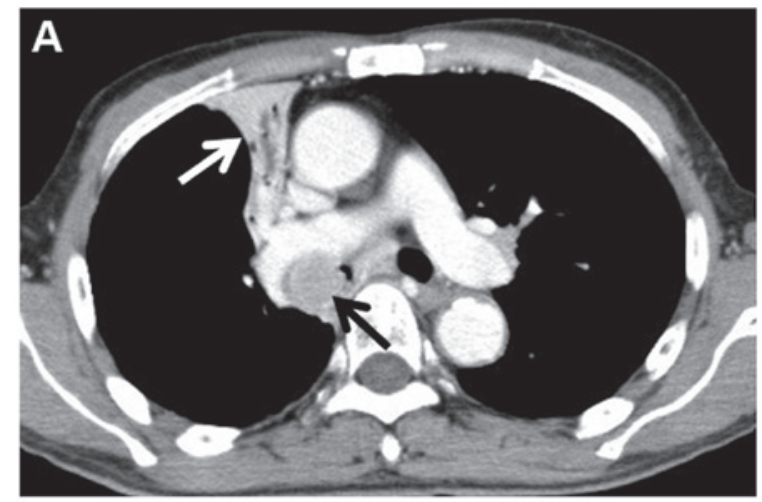

Figure 1. Computed tomography revealed a 26-mm nodule (black arrow) in the right main bronchus and right upper lobe atelectasis (white arrow).

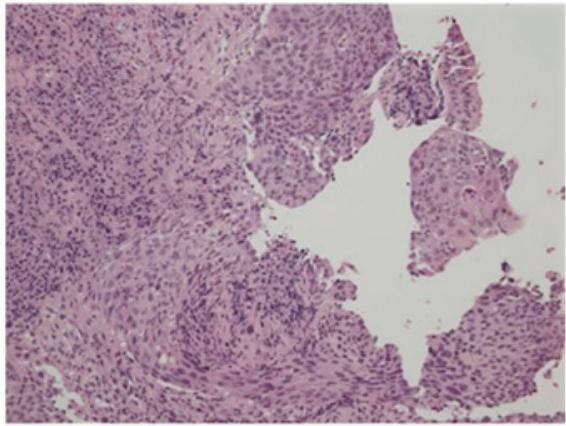

C

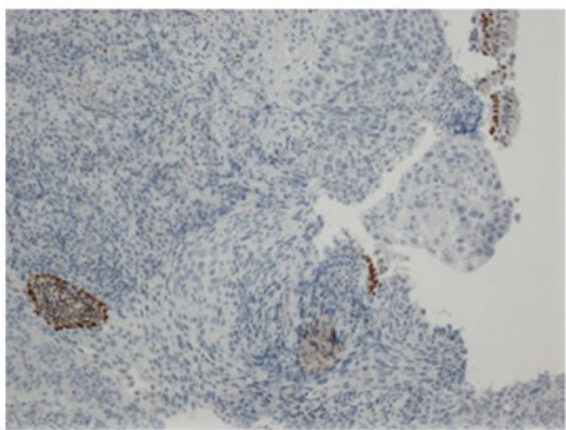

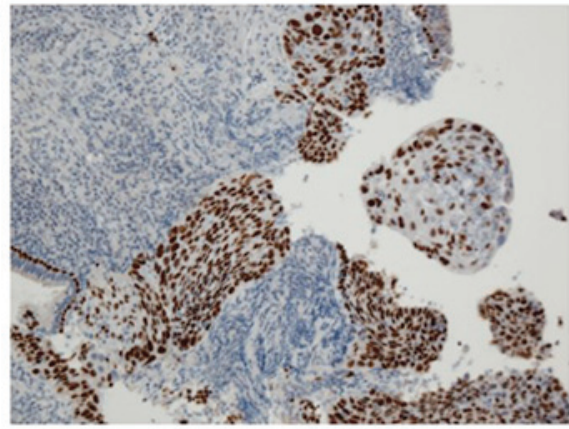

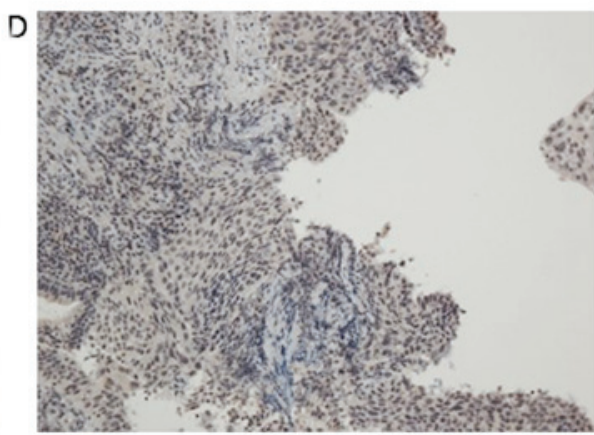

Figure 2. Histopathological and immunohistological findings. (A) Hematoxylin and eosin staining showed squamous cell carcinoma (magnification, $\mathrm{x} 40$ ). (B) Positive immunostaining for $\mathrm{p} 40$ and negative immunostaining for (C) thyroid transcription factor-1 and (D) napsin A.

smokers, younger age at diagnosis, adenocarcinoma histology, signet ring cells, and mutual exclusivity from other major driver genes (1). These clinicopathological characteristics were not applicable to our case, as the patient was a current smoker with hilar-type squamous cell carcinoma. ALK rearrangement in squamous cell carcinoma is extremely rare, with an estimated prevalence of ALK rearrangement in squamous cell carcinoma of the lung of only $\sim 0.2-2.5 \%$ (2). Thus, its molecular analysis when excluding adenocarcinoma is not routinely recommended in the NCCN guideline for the treatment of NSCLC (version 2, 2013).

It is widely known that ALK inhibitors, such as crizotinib or alectinib, have significantly improved treatment response among NSCLC patients with ALK rearrangement. The determination of ALK-positive status is necessary to identify patients with advanced NSCLC who are most likely to benefit from targeted therapy with an ALK inhibitor. The gold standard for the detection of predictive ALK rearrangements is currently break-apart

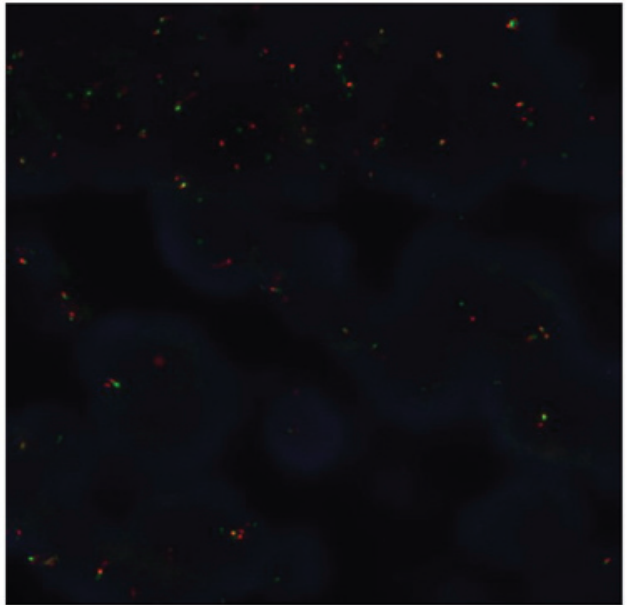

Figure 3. Anaplastic lymphoma kinase (ALK) break-apart fluorescence in situ hybridization showing one fusion signal, and separated red and green signals, revealing ALK rearrangements. The rearrangement-positive cell rate was $20 \%$. 
FISH, as it is able to detect all known ALK rearrangements and was clinically validated in crizotinib clinical trials (3). However, our case was positive for FISH with a $20 \%$ rearrangement-positive cell rate, but negative on IHC. As a possible explanation for this mismatch, the distinction of IHC $1+$ from IHC 0 may be subjective. Re-testing of IHC is desirable; however, there was no residual sample for further testing in this case.

It remains unclear whether ALK-positive squamous cell carcinoma patients show a marked response to ALK-targeted therapies, which is generally effective for ALK-positive lung adenocarcinoma. According to two recent case reports published in China, two 55-year-old female non-smokers with ALK-positive squamous cell carcinoma responded to crizotinib (4,5). By contrast, Tamiya et al reported the case of a 78-year-old male former smoker with ALK-positive squamous cell carcinoma who did not respond to alectinib, a second-generation ALK inhibitor (6), although the tumor cells were confirmed to be diffusely and strongly positive $(3+)$ for ALK on IHC, as well as FISH.

Although this mutation is rare in squamous cell carcinoma, its presence may provide additional treatment options. Our local policy has been to test all patients with advanced NSCLC who may benefit from targeted treatment for activating mutations with sufficient biopsy specimens. In the patient described in this report, subsequent ALK-targeted treatment may be a viable option.

In summary, we reported a case of ALK-positive squamous cell carcinoma of the lung. Oncologist should recognize that ALK translocation may be present in squamous cell carcinoma of the lung, for which targeted therapy may be an effective option.

\section{References}

1. Shaw AT, Yeap BY, Mino-Knudson M, Digumarthy SR, Costa DB, Heist RS, Solomon B, Stubbs H, Admane S, McDermott U, et al: Clinical features and outcome of patients with non-small-cell lung cancer who harbor EML4-ALK. J Clin Oncol 27: 4247-4253, 2009.

2. Caliò A, Nottegar A, Gilioli E, Bria E, Pilotto S, Peretti U, Kinspergher S, Simonato F, Pedron S, Knuutila S, et al: ALK/EML4 fusion gene may be found in pure squamous carcinoma of the lung. J Thorac Oncol 9: 729-732, 2014.

3. Teixido C, Karachaliou N, Peg V, Gimenez-Capitan A and Rosell R: Concordance of IHC, FISH and RT-PCR for EML4-ALK rearrangements. Transl Lung Cancer Res 3: 70-74, 2014.

4. Wang Q, He Y, Yang X, Wang Y and Xiao H: Extraordinary response to crizotinib in a woman with squamous cell lung cancer after two courses of failed chemotherapy. BMC Pulm Med 14: 83, 2014.

5. Zhang Q, Wang J and Zhang S: ALK-rearranged squamous cell lung cancer: A case report. Int J Cjin Exp Pathol 8: 2195-2198, 2015.

6. Tamiya A, Shimizu S and Atagi S: A case of squamous cell carcinoma harboring an EML4/ALK rearrangement that was unsuccessfully treated with the ALK inhibitor alectinib. J Thorac Oncol 10: e74, 2015. 